\title{
Photodynamic inactivation of fibroblasts and inhibition of Staphylococcus epidermidis adhesion and biofilm formation by toluidine blue 0
}

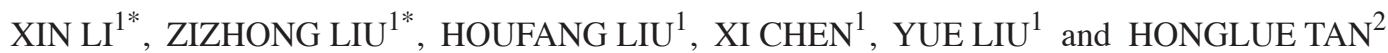 \\ ${ }^{1}$ Department of Dermatology, The Fourth Hospital of Zhenjiang, \\ The Fourth Affiliated Hospital of Jiangsu University, Zhenjiang, Jiangsu 212001; ${ }^{2}$ Department of Orthopedic Surgery, \\ Henan Orthopedic Hospital, Luoyang Orthopedic-Traumatological Hospital, Luoyang, Henan 471002, P.R. China
}

Received December 27, 2015; Accepted December 19, 2016

DOI: $10.3892 / \mathrm{mmr} .2017 .6184$

\begin{abstract}
Treating skin and soft tissue infections of severe limb traumas can be challenging. Crucial concerns focus on inhibiting biofilm formation by antibiotic-resistant bacteria, and preventing scar formation by fibroblastic hyperproliferation. The local use of toluidine blue $\mathrm{O}$ (TBO)-mediated photodynamic therapy (PDT) may be a promising strategy for treating such lesions. The present study used Staphylococcus epidermidis (strain ATCC 35984) to assess the effects of TBO-PDT on bacterial adherence and biofilm formation, using confocal laser scanning microscopy (CLSM), tissue culture plating (TCP) and scanning electron microscopy (SEM). Primary human fibroblast cells were used to evaluate the cytotoxicity of TBO-PDT using the 3-(4,5-dimethylthiazol-2-yl)-2,5-diphenyltetrazolium bromide (MTT) assay and CLSM. Six different treatment groups were investigated: Medium only [tryptone soy broth (TSB) or Dulbecco's modified Eagle's medium (DMEM)]; red light control (light dose, $30 \mathrm{~J} / \mathrm{cm}^{2}$ ); TBO group (50 mM TBO); TBO-PDT1 (TBO irradiated with $10 \mathrm{~J} / \mathrm{cm}^{2}$ ); TBO-PDT2 (TBO irradiated with $20 \mathrm{~J} / \mathrm{cm}^{2}$ ); and TBO-PDT3 (TBO irradiated with $30 \mathrm{~J} / \mathrm{cm}^{2}$ ). The results of the $S$.epidermidis adhesion assay indicated that the TSB, light and TBO groups exhibited significant bacterial
\end{abstract}

Correspondence to: Dr Honglue Tan, Department of Orthopedic Surgery, Henan Orthopedic Hospital, Luoyang Orthopedic-Traumatological Hospital, Luoyang, 82 Qiming South Road, Luoyang, Henan 471002, P.R. China

E-mail: hnlc.love@163.com

Dr Zizhong Liu, Department of Dermatology, The Fourth Hospital of Zhenjiang, The Fourth Affiliated Hospital of Jiangsu University, 20 Zhengdong Road, Zhenjiang, Jiangsu 212001, P.R. China

E-mail: 492151523@qq.com

*Contributed equally

Key words: toluidine blue O, photodynamic therapy, biofilm formation, human fibroblasts adherence, compared with the TBO-PDT groups. Analysis of biofilm formation revealed significant light dose-dependent differences between the TBO-PDT groups and the TSB, light, and TBO groups. Furthermore, SEM indicated fewer colony masses in the TBO-PDT groups compared with the control groups. The MTT assay for fibroblastic cell toxicity demonstrated $\sim 1.1,4.6,14.5,29.7$ and $43.4 \%$ reduction in optical density for the light, TBO, TBO-PDT1, TBO-PDT2 and TBO-PDT3 groups, respectively, compared with the DMEM control group. There was no difference in toxicity between the light and control groups, however, there were significant differences among the TBO-PDT groups. Finally, alterations in fibroblast morphology and cell spreading were revealed by CLSM, following TBO-PDT treatment. TBO-PDT inhibited bacterial adhesion and biofilm formation, and exhibited significant cytotoxic effects on human fibroblasts. These results indicate that the local use of TBO-PDT in limb lesions may be a useful treatment method for inhibiting bacterial biofilm formation and fibroblastic hyperproliferation, which may prevent infectious hypertrophic scar formation.

\section{Introduction}

Skin and soft tissue infection of severe limb traumas is a serious complication that can lead to significant morbidity, and even amputation. Infection rates are correlated with severity of injury, and range between 10 and $50 \%$ for type III open fractures, according to the Gustilo classification (1). Therefore, local infection of soft tissue remains a challenge for plastic surgeons.

Antibiotic-resistant bacteria, and their ability to form biofilms at the trauma site, are a crucial concern. In these cases, sessile bacterial communities become embedded in extracellular polymeric matrices, composed primarily of large polysaccharides, and are adhered via the polysaccharide intercellular adhesin protein $(2,3)$. Once established, biofilms provide a protective niche that promotes bacterial growth and survival. Biofilms are difficult to treat due to the widespread bacterial resistance to antibiotics and host immune defenses (4-6); however, bacteria living in biofilms may be up to 1,000 times more resistant to antibacterial compounds 
than planktonic bacteria (7). Inhibiting bacterial adhesion and proliferation is therefore critical in controlling biofilm formation.

The management of contaminated limb traumas involves repeated debridement of the wound, local and systemic antibiotic application, and two-stage wound closure with free skin grafting or flap grafting. During this procedure, a hypertrophic scar (HS), which is a benign and fibrous overgrowth of scar tissue, results from abnormal wound responses to the trauma. HSs should be avoided, owing to the associated tissue contraction they cause, which subsequently affects limb function and skin aesthetics. The pathological processes of HSs are characterized by fibroblast hyperproliferation (8), and previous research has demonstrated that HS formation can be prevented via physical methods that induce the apoptosis of proliferative fibroblasts (9).

Photodynamic therapy (PDT), which involves the local or systemic administration of a photosensitizer, has recently been used to combat bacterial infections, and studies have shown that planktonic bacterial cells are susceptible to PDT using different photosensitizers (10-12). Toluidine blue O (TBO), which is a cationic phenothiazine dye that has been well studied as an antibacterial photosensitizer, could be used with PDT to inactivate Staphylococcus aureus and S. epidermidis cells by irradiating their biofilms (13-15). However, very few studies have focused on the effects of TBO-PDT on bacterial adhesion and biofilm formation.

PDT may also be an alternative strategy for HS prevention. Cai et al reported that hematoporphyrin monomethyl-mediated PDT induced the apoptosis of fibroblasts isolated from HSs (16), and Chiu et al demonstrated that PDT reduced tissue contraction and collagen density by killing fibroblasts (17). Furthermore, a pilot study by Campbell et al reported that methyl aminolevulinic acid-mediated PDT significantly softened HSs (18). These studies support the hypothesis that TBO-PDT may be useful for HS prevention.

To evaluate these hypotheses, the present study aimed to investigate the potential for TBO-PDT to inhibit bacterial adhesion and biofilm formation, and examined the effect of TBO-PDT on fibroblast proliferation and morphology.

\section{Materials and methods}

Bacterial preparation. S. epidermidis cells (strain: ATCC 35984) were kindly provided by the Shanghai Key Laboratory of Orthopedic Implants (Shanghai, China). This bacterium exhibits a low level of susceptibility to gentamicin [minimum inhibitory concentration $(\mathrm{MIC})=16 \mu \mathrm{g} / \mathrm{ml}$ ] and penicillin ( $\mathrm{MIC}=128 \mu \mathrm{g} / \mathrm{ml}$ ), and has the ability to form biofilms (2). The strain was stored in tryptone soy broth (BD Biosciences, Franklin Lakes, NJ, USA) with 50\% concentration of glycerol at $-80^{\circ} \mathrm{C}$. To obtain inocula for the experiments, strains were cultured on $\mathrm{BBL}^{\mathrm{TM}}$ tryptone soy agar (Shanghai Biotechnology Corporation, Shanghai, China) at $37^{\circ} \mathrm{C}$ for $24 \mathrm{~h}$. A colony was selected and cultured in $10 \mathrm{ml} \mathrm{BBL}^{\mathrm{TM}}$ tryptone soy broth (TSB; BD Biosciences) at $37^{\circ} \mathrm{C}$ for $24 \mathrm{~h}$. Aliquots of $100 \mu \mathrm{l}$ were transferred to sterile tubes containing $10 \mathrm{ml}$ TSB, and incubated at $37^{\circ} \mathrm{C}$ for $12 \mathrm{~h}$ to attain log-phase growth. The bacteria were harvested by centrifugation $(7,000 \times \mathrm{g}$ for $5 \mathrm{~min})$ and were resuspended in sterile phosphate-buffered saline (PBS) to an optical density (OD) of 0.490 , measured at $600 \mathrm{~nm}$, using a Synergy HT Multi-Detection Microplate Reader spectrophotometer (BioTek Instruments Inc., Winooski, VT, USA). The resulting suspension contained $10^{9}$ colony forming units $(\mathrm{CFU}) / \mathrm{ml}$. Prior to each experiment, the cells were diluted in $\mathrm{TSB}$ to the requisite density of $10^{7} \mathrm{CFU} / \mathrm{ml}$.

Cell preparation. L929 mouse fibroblast cells were kindly provided by the Shanghai 9th People's Hospital (Shanghai, China). Cells were suspended in high-glucose Dulbecco's modified Eagle's medium (DMEM; Invitrogen; Thermo Fisher Scientific, Inc., Waltham, MA, USA) supplemented with $10 \%$ fetal bovine serum (HyClone; GE Healthcare Life Sciences, Logan, UT, USA), $100 \mathrm{IU} / \mathrm{ml}$ penicillin, and $100 \mu \mathrm{g} / \mathrm{ml}$ streptomycin (both Sigma-Aldrich; Merck Millipore, Darmstadt, Germany). The cells were then seeded in 6-well culture plates (Corning Incorporated, New York, NY, USA) at a density of $3 \times 10^{5}$ cells/well, and were maintained in a humidified incubator at $37^{\circ} \mathrm{C}$ with $5 \% \mathrm{CO}_{2}$. Cells were passaged every 3 days until the cell monolayer reached $>80 \%$ confluence. The growth medium was replaced every day. Cells obtained from passages 2 and 3 were used for analysis.

PDT and experimental groups. A stock solution of TBO (Sigma-Aldrich; Merck Millipore) was prepared immediately prior to use, in distilled water. TBO was subsequently filter sterilized, and kept in the dark at $4^{\circ} \mathrm{C}$ until use. The TBO dye was then diluted with sterile TSB or DMEM to $50 \mathrm{mM}$, a concentration that was chosen based on previous research $(13,15,19,20)$. TBO irradiation was performed at 4 different light doses, using a wavelength of $635 \mathrm{~nm}$, on a HPG5000 semiconductor laser (Elforlight, Daventry, UK). The experimental setup was as follows: Medium control group (TSB or DMEM without TBO or light); light control group (TSB or DMEM; light dose, $30 \mathrm{~J} / \mathrm{cm}^{2}$, without TBO); TBO control, (light dose, $0 \mathrm{~J} / \mathrm{cm}^{2}$ ); TBO-PDT1 (light dose, $10 \mathrm{~J} / \mathrm{cm}^{2}$ ); TBO-PDT2 (light dose, $20 \mathrm{~J} / \mathrm{cm}^{2}$ ); TBO-PDT3 (light dose, $30 \mathrm{~J} / \mathrm{cm}^{2}$ ).

Analysis of adhesion by confocal laser scanning microscopy (CLSM). Bacteria were resuspended at a density of $1.0 \times 10^{7} \mathrm{CFU} / \mathrm{ml}$, in TSB containing $50 \mathrm{mM}$ TBO. Glass coverslips were placed into the wells of a 24-well Costar 3548 microtiter plate (Corning Incorporated), $2 \mathrm{ml}$ bacterial cells were seeded, and the plate was incubated at $37^{\circ} \mathrm{C}$ in the dark, for $30 \mathrm{~min}$, with agitation $(100 \mathrm{rpm})$. The TBO-PDT groups were irradiated at different light doses $\left(0,10,20\right.$ and $\left.30 \mathrm{~J} / \mathrm{cm}^{2}\right)$, and the light control group was irradiated with a light dose of $30 \mathrm{~J} / \mathrm{cm}^{2}$. Laser light was focused on a $2.0 \mathrm{~cm}^{2}$ spot using a special diffuse optical fiber (HPG5000 laser; wavelength, $635 \mathrm{~nm}$; Elforlight Ltd, Daventry, UK). The culture suspensions were then transferred to a new plate containing sterile coverslips and cultured at $37^{\circ} \mathrm{C}$ for $4 \mathrm{~h}$.

To assess bacterial adhesion, the coverslips were subsequently transferred into a fresh 24-well plate and gently washed with PBS 3 times to remove loosely adherent bacteria. All coverslips with attached cells were stained using the Live/Dead Baclight Bacterial Viability kit (Molecular Probes; Thermo Fisher Scientific, Inc., Waltham, MA, USA) and incubated for $15 \mathrm{~min}$ in the dark. The viability kit includes two fluorescent nucleic acid stains: SYTO9 and 
propidium iodide (PI). Dead cells take up PI and fluoresce red, whereas live cells take up SYTO9 and fluoresce green. Cell regions were selected randomly, and analyzed by CLSM using a Leica TCS SP2 (Leica Microsystems GmbH, Wetzlar, Germany).

Analysis of biofilm formation by tissue culture plating (TCP). The TCP method is regarded as the standard test in assessing biofilm formation (2). Bacterial cells were prepared and PDT was performed as described in the aforementioned bacterial adhesion assay analysis, with the exception that the cells were adhered onto 96-well microtiter plates (laser light was focused on $\left.0.32-\mathrm{cm}^{2} \mathrm{spot}\right)$. Bacterial suspensions $(200 \mu \mathrm{l})$ were transferred to new 96 -well plates and incubated at $37^{\circ} \mathrm{C}$ in the dark, for $24 \mathrm{~h}$. The next day, biofilms that had formed in the bottom of the plates were washed 3 times with PBS to remove planktonic cells. The plates were dried at $60^{\circ} \mathrm{C}$ for $1 \mathrm{~h}$, and adherent bacteria were fixed with $95 \%$ ethanol for $10 \mathrm{~min}$ and stained at room temperature with $200 \mu 10.1 \%$ crystal violet (CV) for $15 \mathrm{~min}$. The plates were rinsed 3 times with PBS to remove excess stain. The plates were then dried at $37^{\circ} \mathrm{C}$ for $2 \mathrm{~h}$, and biofilm formation was quantified by solubilization of the CV stain in $200 \mu 130 \%$ (w/v) glacial acetic acid for 15 min with agitation (300 rpm). The concentration of $\mathrm{CV}$ was determined by measuring the absorbance at $492 \mathrm{~nm}$ using a microplate reader. The mean absorbance value obtained from the medium control well was deducted from the test absorbance values, and the values were expressed as optical density $\left(\mathrm{OD}_{492}\right)$. An $\mathrm{OD}_{492}$ value $<0.120$ suggested that no biofilm was formed. Moderate and high biofilm formations were detected when $\mathrm{OD}_{492}$ values ranged between 0.120 and 0.240 , or were $>0.240$, respectively $(21,22)$.

Analysis of biofilm formation by scanning electron microscopy (SEM). Following PDT as aforementioned, plates with coverslips were incubated for $24 \mathrm{~h}$ at $37^{\circ} \mathrm{C}$, the culture medium was removed, and the coverslips were washed 3 times with PBS to remove non-adherent bacteria. All the coverslips with biofilms were fixed in $2.5 \%$ glutaraldehyde for $2 \mathrm{~h}$ at $4^{\circ} \mathrm{C}$, and then dehydrated via a series of increasing ethanol solutions $(25,50,75,95$ and $100 \%)$. The samples were subsequently freeze-dried, sputter-coated with gold, and observed using a JEOL JSM-6310LV scanning electron microscope (JEOL, Ltd., Tokyo, Japan).

Analysis of cell toxicity by 3-(4,5-dimethylthiazol-2-yl)-2,5-diphenyltetrazolium bromide (MTT). Fibroblasts were seeded into a 96-well plate at a density of $1.5 \times 10^{3}$ cells per $100 \mu \mathrm{l}$ of DMEM containing $50 \mathrm{mM} \mathrm{TBO}$, and then incubated, at $37^{\circ} \mathrm{C}$ with $5 \% \mathrm{CO}_{2}$, in the dark, for $30 \mathrm{~min}$. TBO-PDT groups were irradiated with a $635 \mathrm{~nm}$ laser at different light doses $(0,10$, 20 or $30 \mathrm{~J} / \mathrm{cm}^{2}$ ). Laser light was focused on a $0.32-\mathrm{cm}^{2}$ spot using an appropriate diffuse optical fiber. The media was then exchanged for fresh DMEM (100 $\mu \mathrm{l})$ and the cells were incubated overnight at $37^{\circ} \mathrm{C}$, followed by a 24-h incubation in the dark. Cell toxicity was determined using the MTT assay. Briefly, $20 \mu 1$ MTT (Sigma-Aldrich; Merck Millipore) was added to each well, and the plates were incubated at room temperature for $4 \mathrm{~h}$. The MTT-containing medium was then removed, and the formazan crystals were solubilized in $200 \mu \mathrm{l}$ dimethyl sulfoxide (Sigma-Aldrich; Merck Millipore). The OD was measured at $570 \mathrm{~nm}$ using a microplate reader.

Analysis of cell morphology by CLSM. Fibroblast cells were seeded into 48-well plates containing coverslips at a density of $1 \times 10^{4}$ cells $/ \mathrm{ml}$, and cultured in DMEM containing $50 \mathrm{mM}$ TBO. The plates were incubated at $37^{\circ} \mathrm{C}$ with $5 \% \mathrm{CO}_{2}$, in the dark, for $30 \mathrm{~min}$. The TBO-PDT groups were irradiated with a $635 \mathrm{~nm}$ laser at different light doses $\left(0,10,20\right.$ or $\left.30 \mathrm{~J} / \mathrm{cm}^{2}\right)$. Laser light was focused on a $0.8-\mathrm{cm}^{2}$ spot using an appropriate diffuse optical fiber. The plates were then incubated at $37^{\circ} \mathrm{C}$ with $5 \% \mathrm{CO}_{2}$, in the dark, for $24 \mathrm{~h}$. The next day, coverslips were transferred to a new plate and washed three times with PBS, and then fixed using 3.7\% formaldehyde for $15 \mathrm{~min}$ at room temperature. The cells were permeabilized with $0.1 \%$ Triton X-100 in PBS for another $15 \mathrm{~min}$. Following three washes with PBS, the cells were incubated with fluorescein rhodamine phalloidin (20 mg/ml; Sigma-Aldrich; Merck Millipore) for $1 \mathrm{~h}$. Following another PBS wash, the cell nuclei were stained with DAPI $(1 \mathrm{mg} / \mathrm{ml})$, resulting in red and blue fluorescence, respectively. Cell regions were selected randomly and analyzed by CLSM, and cell spreading was quantified using an image analyzer (ImageJ; version 1.48; National Institutes of Health, Bethesda, MD, USA).

Statistical analysis. All experiments were performed in triplicate and repeated three times. The data are presented as the mean \pm standard deviation. The results were tested using a one-way analysis of variance and Tukey's multiple comparison tests. Statistical analyses were performed using the SAS analysis software (version, 9.4; SAS Institute Inc., Cary, NC, USA). $\mathrm{P}<0.05$ was considered to indicate a statistically significant difference.

\section{Results}

Irradiated TBO inhibits bacterial adhesion. Representative CLSM images of $S$. epidermidis (ATCC 35984) in the adhesion assay are shown in Fig. 1. The greatest intensity of green fluorescence was observed in the TSB control, light and TBO groups (Fig. 1A-C), indicating a significant amount of bacterial adherence. The green fluorescence intensities were markedly reduced in the TBO-PDT1-3 groups, demonstrating that irradiated TBO was effective in inhibiting bacterial adhesion in a light dose-dependent manner.

Irradiated TBO inhibits biofilm formation. The $\mathrm{OD}_{492}$ values for ATCC 35984 are shown in Fig. 2. No differences were detected among the TSB control, light and TBO groups $(P>0.05)$. The absorbance values decreased gradually with increasing light dose in the TBO-PDT1-3 groups, which was significantly different compared with the TSB, light and TBO groups $(\mathrm{P}<0.01)$. The differences among the three TBO-PDT groups were also significant $(\mathrm{P}<0.05)$. The values of the TBO-PDT groups did not exceed the lower OD limit of 0.120 , a value indicating the presence of the biofilm-producing bacterial strain (22), indicating that irradiated TBO inhibited biofilm formation of ATCC 35984.

SEM images are presented in Fig. 3. Large cellular aggregates surrounded by extracellular matrix were observed 


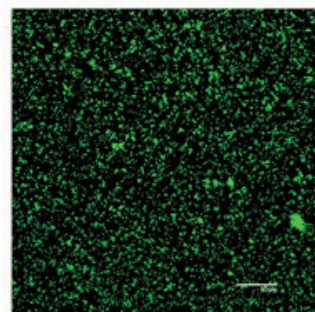

A

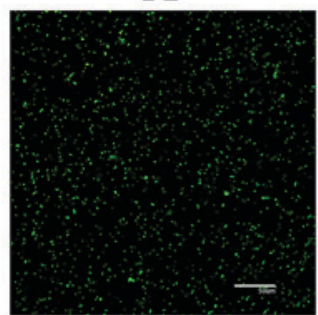

D

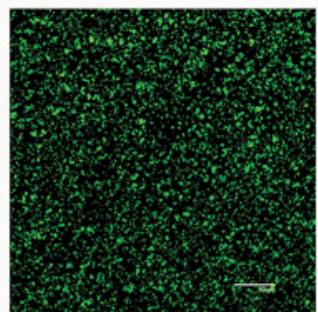

B

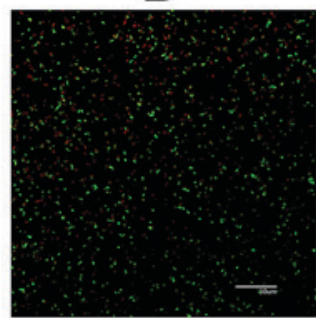

$\mathrm{E}$

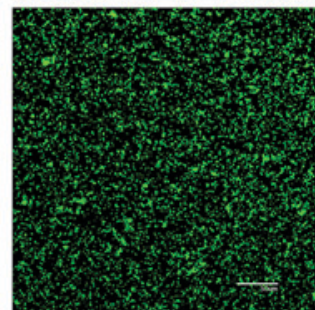

C

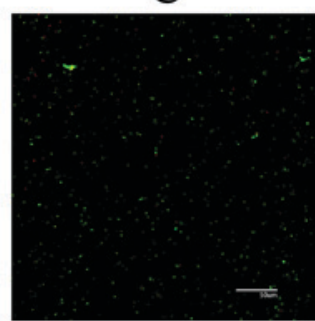

$\mathrm{F}$

Figure 1. Confocal laser scanning microscopy images of ATCC 35984 adhesion. (A) Trytpone soy broth group, (B) light group, (C) TBO group, (D) TBO-PDT1 group, (E) TBO-PDT2 group and (F) TBO-PDT3 group. Magnification, x200. Scale bar, $50 \mu \mathrm{m}$. ATCC 35984, Staphylococcus epidermidis cells; TBO, toluidine blue O; PDT, photodynamic therapy.

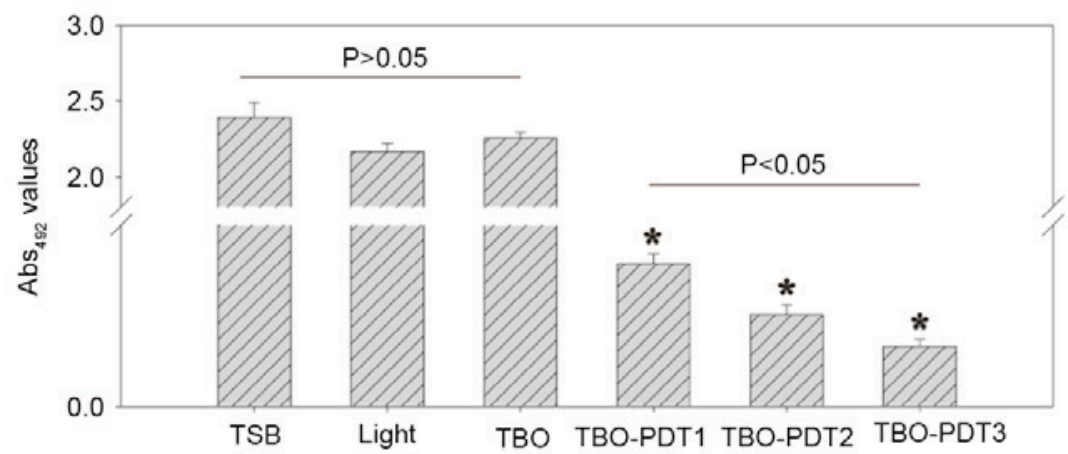

Figure 2. Biofilm assay for ATCC 35984, as determined by tissue culture plating. ${ }^{*} \mathrm{P}<0.01$, compared with TSB, light and TBO groups. The differences were significant among the TBO-PDT groups ("P<0.05). ATCC 35984, Staphylococcus epidermidis cells; TSB, tryptone soy broth; TBO, toluidine blue O; PDT, photodynamic therapy.

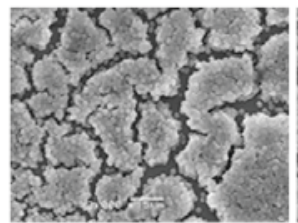

A

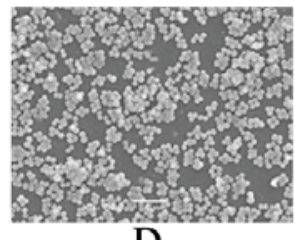

D

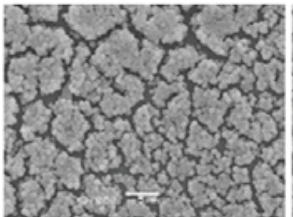

B

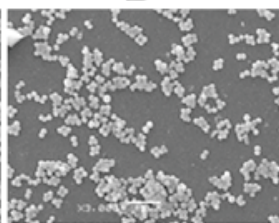

$\mathrm{E}$

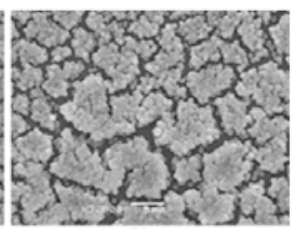

$\mathrm{C}$

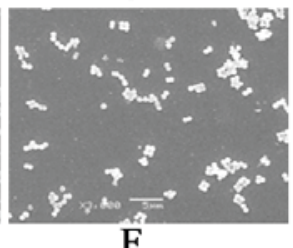

Figure 3. Scanning electron microscopy images of ATCC 35,984 biofilm formation. (A) Tryptone soy broth group, (B) light group, (C) TBO group,(D) TBO-PDT1 group, (E) TBO-PDT2 group and (F) TBO-PDT3 group. Magnification, x3,000. Scale bar, $5 \mu \mathrm{m}$. ATCC 35984, Staphylococcus epidermidis cells; TBO, toluidine blue O; PDT, photodynamic therapy.

in the TSB, light and TBO groups, indicating biofilm formation (Fig. 3A-C). In the TBO-PDT groups, the number and size of colony masses decreased with increasing light dose,
(Fig. 3D-F). In the TBO-PDT3 group, a few aggregated colonies were observed in isolation, indicating an absence of biofilm formation (Fig. 3F). 


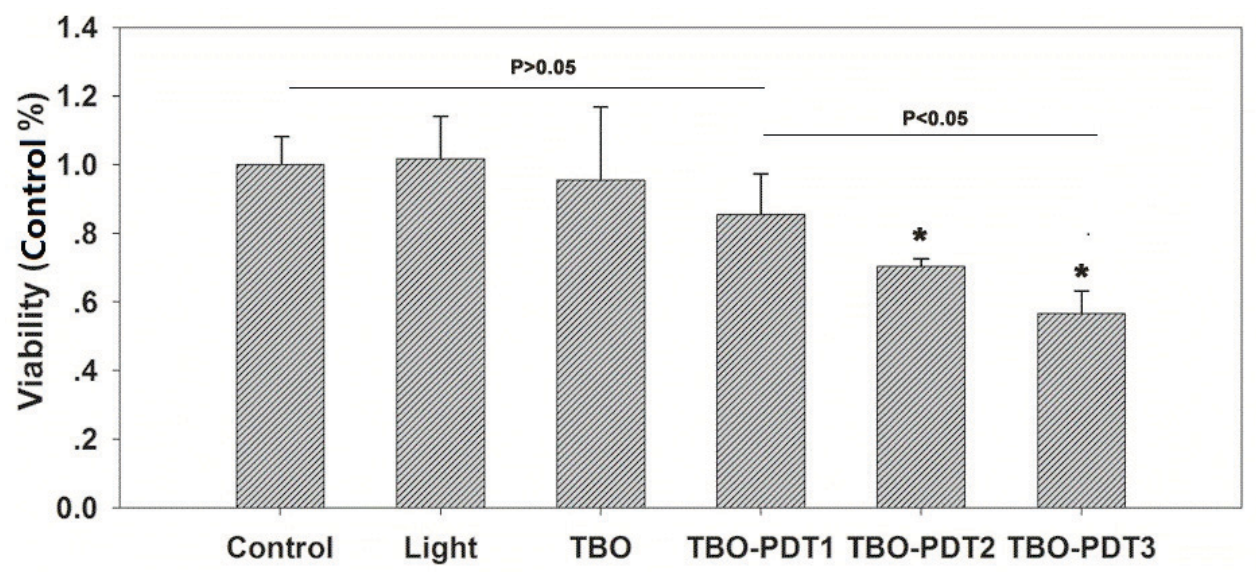

Figure 4. Fibroblast cytotoxicity after TBO-PDT treatment, as determined by MTT assay. "P<0.05, compared with the Dulbecco's modified Eagle's medium control, light, TBO and TBO-PDT1 groups. TBO, toluidine blue O; PDT, photodynamic therapy.
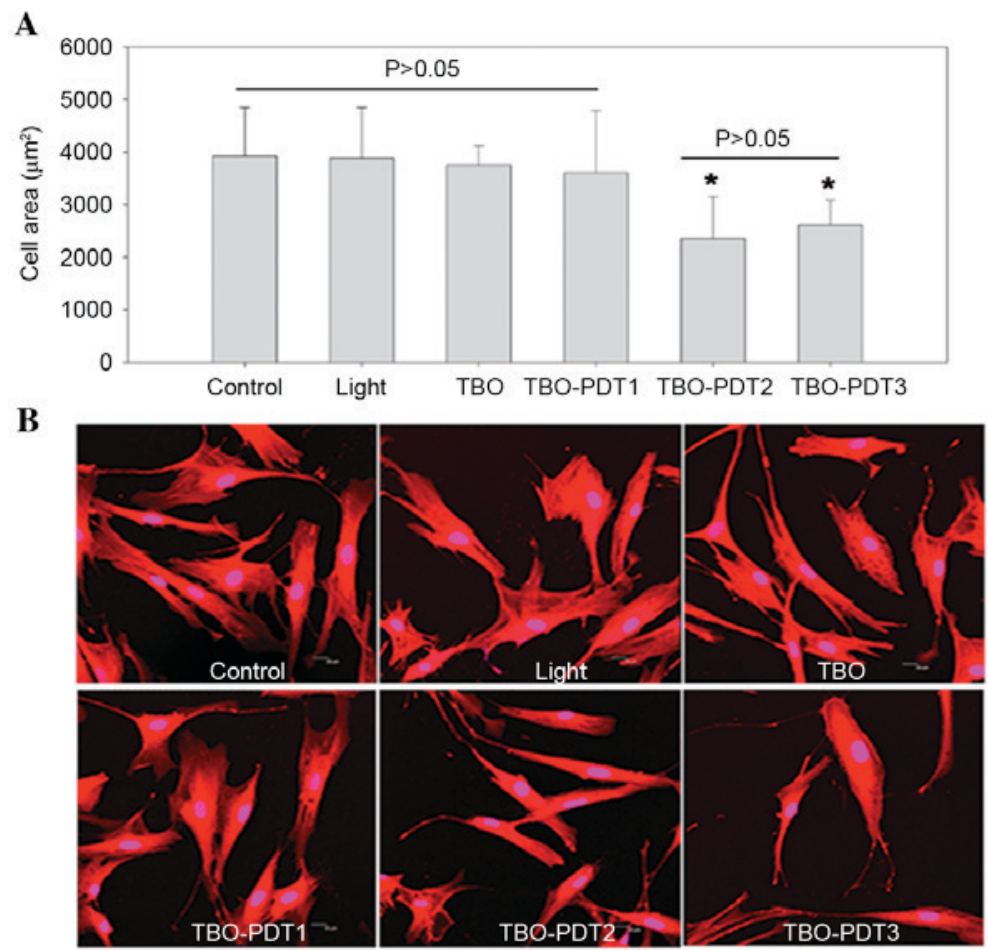

Figure 5. Morphology of TBO-PDT treated primary human fibroblasts by confocal laser scanning microscopy. (A) The area of cell spreading in different treatment groups. "P<0.05, compared with the Dulbecco's modified Eagle's medium, light, TBO and TBO-PDT1 groups. (B) Images of cytoskeletal morphology. Cells were stained with rhodamine phalloidin for actin filaments (red) and nuclei were counterstained with DAPI (blue). Magnification, x400. TBO, toluidine blue O; PDT, photodynamic therapy.

Irradiated TBO induces toxicity of fibroblasts. Analysis of fibroblast cells by MTT assay induced a 1.1, 4.6, 14.5, 29.7 and $43.4 \%$ reduction in optical density for the light, TBO, TBO-PDT1, TBO-PDT2, and TBO-PDT3 groups respectively, compared with the DMEM (control) group (Fig. 4); however, there was no difference in toxicity between the control and light groups. Differences between the TBO-PDT1, TBO-PDT2, and TBO-PDT3 groups were statistically significant $(\mathrm{P}<0.05)$. There was also a significant $(\mathrm{P}<0.05)$ reduction in optical density between the TBO-PDT2 and TBO-PDT3 groups, compared with the control, light, TBO, and TBO-PDT1 groups. A slight but non-statistically significant decrease in optical density was observed in the TBO-PDT1 group compared with the DMEM, light and TBO groups $(\mathrm{P}>0.05)$. No significant toxicity was observed in the light or TBO control groups, compared with the DMEM control group $(\mathrm{P}>0.05)$.

Irradiated TBO reduces cell spreading. The areas of cell spreading in the control, light, TBO, TBO-PDT1, TBO-PDT2 and TBO-PDT3 groups were determined to be $3,922.25 \pm 923.64,3,880.5 \pm 968.21,3,750.25 \pm 369.68$, $3,607.5 \pm 1185.28,2,357.5 \pm 795.75$ and $2,619.5 \pm 468.62 \mu \mathrm{m}^{2}$, respectively (Fig. 5A). There were no statistically significant differences among the control, light, TBO and TBO-PDT1 groups. The TBO-PDT2 and TBO-PDT3 groups exhibited smaller areas of cell spreading compared with the DMEM, 
light, TBO and TBO-PDT1 groups $(\mathrm{P}<0.05)$, however no difference was found between the TBO-PDT2 and TBO-PDT3 groups. Inspection of the CLSM images revealed that the level of cell spreading in the control, light, TBO and TBO-PDT1 groups was similar (Fig. 5B), and the cells exhibited a polygonal and fusiform-shaped morphology. However the cells in the TBO-PDT2 and TBO-PDT3 groups predominantly exhibited a fusiform-shaped morphology.

\section{Discussion}

PDT is a promising strategy to combat biofilm formation by bacteria. Previous studies have described TBO-PDT-based anti-biofilm strategies against various microbial species (10,13-15). However, the evaluation of the potential of TBO-PDT to inhibit bacterial adhesion and biofilm formation, particularly by antibiotic-resistant bacteria, has received little attention.

The present study investigated the effects of TBO-PDT on adhesion and biofilm formation by S.epidermidis strain ATCC 35984. It was demonstrated that neither treatment with TBO nor irradiation with red light, in isolation, affected the adhesion and biofilm formation of strain ATCC 35984. The number of adherent cells of ATCC 35984 was significantly reduced when the cells were irradiated with red light in the presence of TBO, and the number of adherent cells decreased with increasing light doses. These results indicated that TBO-PDT may inhibit bacterial adhesion, a process that is essential for biofilm formation.

TCP analysis was used to further evaluate the effectiveness of TBO-PDT on biofilm formation. The $\mathrm{OD}_{492}$ values of ATCC 35984 were higher in the TSB, TBO and light groups; however, these values were reduced when the bacteria were irradiated with red light in the presence of TBO. Notably, the optical density value was $<0.120$ at the higher light doses of 20 or $30 \mathrm{~J} / \mathrm{cm}^{2}$, indicating that no biofilms were formed. Imaging by SEM supported these results, where it was revealed that ATCC 35984 had formed extensive biofilms in the TSB, TBO and light groups; however, the number and size of colony masses decreased in the TBO-PDT groups with increasing light doses. In the TBO-PDT2 and TBO-PDT3 groups, there were very few single-layered small colonies, suggesting that biofilms were not present. These data therefore indicated that TBO-PDT may lead to cell detachment, resulting in inhibited biofilm formation.

For severe limb traumas with contamination and soft tissue defects, debridement of the wound is usually required prior to closing the wound. The risk of infection is very high at this point, and it is at this stage that the scar-forming HSs may form, from the hyperproliferation of fibroblasts. An efficient method to prevent scar formation would involve inhibition of fibroblast proliferation; a few approaches to this have previously been researched $(19,23,24)$. The present study investigated the photodynamic effects of TBO with red light irradiation on fibroblasts. MTT assay analysis revealed light dose-dependent reductions in optical density for the TBO-PDT1, TBO-PDT2 and TBO-PDT3 groups, compared with the DMEM control group; these decreases were significant for the TBO-PDT2 and TBO-PDT3 groups. These results indicated that TBO-PDT induced significant cytotoxic effects on in vitro cultured fibroblasts. Furthermore, double fluorescence cell staining CLSM was used to observe that the fibroblastic cellular morphologies in TBO-PDT2 and TBO-PDT3 groups mainly exhibited a fusiform-shaped morphology. The adherent areas were significantly decreased, which may have been caused by the toxicity of irradiated TBO to the fibroblasts. These findings indicated that the activity of the fibroblasts was impaired under TBO-PDT treatment, and suggested that TBO-mediated PDT may be useful for HS prevention.

In conclusion, the present study demonstrated that TBO-PDT inhibits bacterial adhesion and biofilm formation, whilst inducing significant cytotoxic effects on human fibroblasts. TBO-PDT may be a useful method for inhibiting bacterial adhesion, biofilm formation and HS formation; limb traumas with serious bacterial contamination and soft tissue defects may therefore benefit from local treatment with TBO-PDT. These findings should be further investigated using in vivo studies.

\section{Acknowledgements}

This study was supported by the Fourth Batch of Science and Technology Plan of Zhenjiang, Jiangsu, China (grant no. SH2015063).

\section{References}

1. Gustilo RB and Anderson JT: Prevention of infection in the treatment of one thousand and twenty-five open fractures of long bones: Retrospective and prospective analyses. J Bone Joint Surg Am 58: 453-458, 1976.

2. Tan HL, Peng ZX, Li QT, Xu XF, Guo SR and Tang TT: The use of quaternised chitosan-loaded PMMA to inhibit biofilm formation and downregulate the virulence-associated gene expression of antibiotic-resistant staphylococcus. Biomaterials 33: 365-377, 2012.

3. Chokr A, Watier D, Eleaume H, Pangon B, Ghnassia JC, Mack D and Jabbouri S: Correlation between biofilm formation and production of polysaccharide intercellular adhesin in clinical isolates of coagulase-negative staphylococci. Int J Med Microbiol 296: 381-388, 2006.

4. Stewart PS and Costerton JW: Antibiotic resistance of bacteria in biofilms. Lancet 358: 135-138, 2001.

5. Begun J, Gaiani JM, Rohde H, Mack D, Calderwood SB, Ausubel FM and Sifri CD: Staphylococcal biofilm exopolysaccharide protects against Caenorhabditis elegans immune defenses. PLoS Pathog 3: e57, 2007.

6. Kristian SA, Birkenstock TA, Sauder U, Mack D, Götz F and Landmann R: Biofilm formation induces $\mathrm{C} 3$ a release and protects Staphylococcus epidermidis from IgG and complement deposition and from neutrophil-dependent killing. J Infect Dis 197: 1028-1035, 2008.

7. Montanaro L, Campoccia D and Arciola CR: Advancements in molecular epidemiology of implant infections and future perspectives. Biomaterials 28: 5155-5168, 2007.

8. Gauglitz GG, Korting HC, Pavicic T, Ruzicka T and Jeschke MG: Hypertrophic scarring and keloids: Pathomechanisms and current and emerging treatment strategies. Mol Med 17: 113-125, 2011.

9. Vrijman C, van Drooge AM, Limpens J, Bos JD, van der Veen JP, Spuls PI and Wolkerstorfer A: Laser and intense pulsed light therapy for the treatment of hypertrophic scars: A systematic review. Br J Dermatol 165: 934-942, 2011.

10. Jori G: Photodynamic therapy of microbial infections: State of the art and perspectives. J Environ Pathol Toxicol Oncol 25: 505-519, 2006.

11. Tang HM, Hamblin MR and Yow CM: A comparative in vitro photoinactivation study of clinical isolates of multidrug-resistant pathogens. J Infect Chemother 13: 87-91, 2007.

12. Wainwright M: Photoantimicrobials-so what's stopping us? Photodiag Photodyn Ther 6: 167-169, 2009. 
13. Zanin IC, Gonçalves RB, Junior AB, Hope CK and Pratten J: Susceptibility of Streptococcus mutans biofilms to photodynamic therapy: An in vitro study. J Antimicrob Chemother 56 324-330, 2005.

14. Zanin IC, Lobo MM, Rodrigues LK, Pimenta LA, Höfling JF and Goncalves LB: Photosensitization of in vitro biofilms by toluidine blue $\mathrm{O}$ combined with light-emitting diode. Eur J Oral Sci 114: 64-69, 2006.

15. Sharma M, Visai L, Bragheri F, Cristiani I, Gupta PK and Speziale P: Toluidine blue-mediated photodynamic effects on staphylococcal biofilms. Antimicrob Agents Chemother 52: 299-305, 2008.

16. Cai H, Gu Y, Sun Q, Zeng J, Dong N and Zhao G: Effect of hematoporphyrin monomethyl ether-mediated photodynamic therapy on hypertrophic scar fibroblasts. Photodermatol Photoimmunol Photomed 27: 90-96, 2011.

17. Chiu LL, Sun CH, Yeh AT, Torkian B, Karamzadeh A, Tromberg B and Wong BJ: Photodynamic therapy on keloid fibroblasts in tissue-engineered keratinocyte-fibroblast co-culture. Lasers Surg Med 37: 231-234, 2005.

18. Campbell SM, Tyrrell J, Marshall R and Curnow A: Effect of MAL-photodynamic therapy on hypertrophic scarring. Photodiagnosis Photodyn Ther 7: 183-188, 2010.

19. Kashef N, Ravaei Sharif Abadi G and Djavid GE: Photodynamic inactivation of primary human fibroblasts by methylene blue and toluidine blue O. Photodiagnosis Photodyn Ther 9: 355-358, 2012.
20. Sahu K, Bansal H, Mukherjee C, Sharma M and Gupta PK: Atomic force microscopic study on morphological alterations induced by photodynamic action of Toluidine Blue $\mathrm{O}$ in Staphylococcus aureus and Escherichia coli. J Photochem and Photobiol B 96: 9-16, 2009.

21. Christensen GD, Simpson WA, Younger JJ, Baddour LM, Barrett FF, Melton DM and Beachey EH: Adherence of coagulase-negative staphylococci to plastic tissue culture plates: A quantitative model for the adherence of staphylococci to medical devices. J Clin Microbiol 22: 996-1006, 1985.

22. Dosselli R, Millioni R, Puricelli L, Tessari P, Arrigoni G, Franchin C, Segalla A, Teardo E and Reddi ED: Molecular targets of antimicrobial photodynamic therapy identified by a proteomic approach. J Proteomics 77: 329-343, 2012.

23. Gupta S and Sharma VK: Standard guidelines of care: Keloids and hypertrophic scars. Indian J Dermatol Venereol Leprol 77: 94-100, 2011.

24. Berman B, Perez OA, Konda S, Kohut BE, Viera MH, Delgado S, Zell D and Li Q: A review of the biologic effects, clinical efficacy, and safety of silicone elastomer sheeting for hypertrophic and keloid scar treatment and management. Dermatol Surg 33: 1291-1303, 2007. 